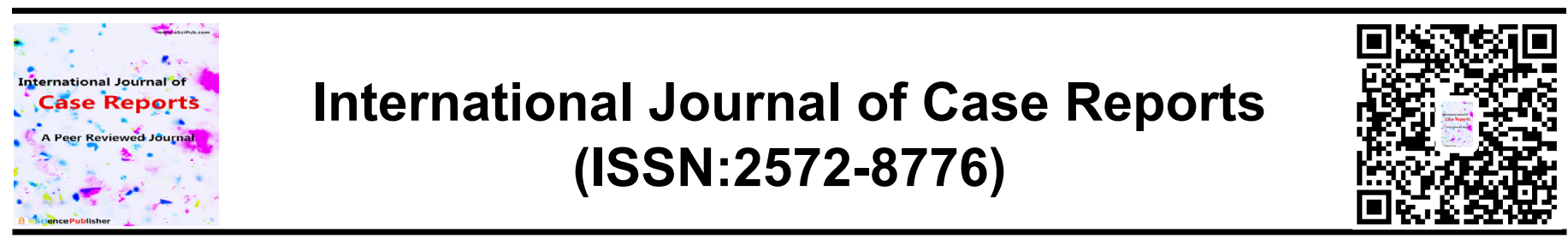

\title{
Congenital Bilateral Microtia-Anotia: A Clinical Challenge
}

\section{Dr Sumita Mehta ${ }^{1^{*}}$, Senior Specialist \& In-Charge, Dr Anshul Grover ${ }^{1}$, Specialist, Dr Avdesh Mehta $^{2}$, ENT Consultant, Dr Deepak Chugh ${ }^{3}$, CMO \& Head of Department, Paediatrics}

${ }^{1}$ Dept. of Obstetrics \& Gynecology, Babu Jagjivanram Memorial Hospital, Delhi, India.

${ }^{2}$ Medhealers Clinic, Delhi, India.

${ }^{3}$ Dept. of Pediatrics, Babu Jagjivanram Memorial Hospital, Delhi, India.

\section{ABSTRACT}

Microtia- Anotia (M-A) is a rare congenital malformation of the external ear structure commonly associated with atresia or stenosis of the ear canal. Its prevalence is more common in Asians and Hispanics, is generally unilateral and is seen more commonly in males. It can either be an isolated defect or be a part of specific pattern of anomalies involving other organs. It has been documented as a component of either a teratogenic embryopathy (isotretinoin, thalidomide or alcohol) or single gene disorders like Treacher Collins and as a part of a pattern of multiple defects like Goldenhar, Nager and CHARGE syndrome. We report a case of a newborn with bilateral microtia-anotia and facial palsy, who on further evaluation was found to have congenital heart disease also. The triad of anotia, facial paralysis and cardiac anomaly is rare and has been first described by Pearl in 1984.Thus, the obviously visible ear malformation at birth should be evaluated in depth to rule out other underlying malformations.

Keywords: microtia, anotia, Pearl syndrome, Goldenhar syndrome, lower motor neuron facial palsy, Treacher Collins syndrome, Nager syndrome, CHARGE syndrome
${ }^{*}$ Correspondence to Author:

Dr Sumita Mehta

Senior Specialist, BJRM Hospital, Delhi, India-110033

How to cite this article:

Sumita Mehta, Anshul Grover, Avdesh Mehta, Deepak Chugh. Congenital Bilateral MicrotiaAnotia: A Clinical Challenge. International Journal of Case Reports, 2021; 5:221.

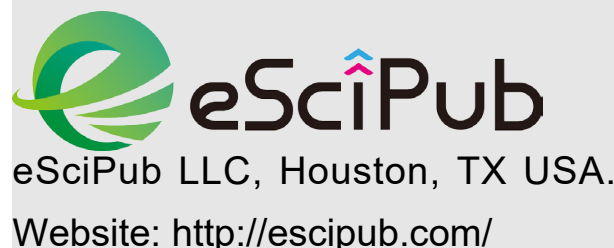

Website: http://escipub.com/ 


\section{Introduction}

Microtia or anotia are auricular development anomalies which are relatively rare with prevalence estimates varying between 0.83 to $17.4 \%$ per 10,000 live births among various countries. They are seen more commonly in males (20$40 \%$ increased risk), unilateral involvement is seen in $77-93 \%$ patients with higher involvement of the right ear $(60 \%)$. ${ }^{[1,2]}$ They are part of a spectrum of first and second branchial arch abnormalities and can present as isolated defects or be associated with various other syndromes. [3] The risk factors for development of microtia include higher maternal age, higher parity, multiple births, maternal diabetes, low birth weight and maternal use of medications like thalidomide and retinoids. Anotia which is apparent at birth should alert the treating neonatologist to further evaluate the infant to exclude congenital anomalies of distant organs. We report successful management of an infant who was born with congenital bilateral microtia-anotia and right facial nerve motor weakness and who on further evaluation was found to have associated acyanotic heart disease.

\section{Case Discussion}

A 26 year old, second gravida presented at 38 weeks of gestation to our hospital in advanced active labor with meconium stained liquor and delivered a $2.8 \mathrm{~kg}$ male baby vaginally. She had only two previous antenatal visits, one at 16 weeks and the other at 32 weeks of gestation. Her antenatal blood investigations including diabetes screen was normal. Only one level I ultrasound was performed at 16 weeks, which did not suggest any anomaly. It was a consanguineous marriage and she was married to her second degree cousin. The mother did not suffer from any chronic illnesses and had a previous 5 year old healthy female child delivered vaginally at term. Detailed antenatal history of the mother suggested that she had not taken iron or folic acid supplements during her pregnancy. There was no history suggestive of any intrauterine infection, radiation exposure, or any drug intake.

The newborn did not cry immediately at birth and required tactile stimulation and oxygen support. There was no apnea, grunting or chest retraction and APGAR score at 1 and 5 minutes was 7 and 8 respectively. On examination, the newborn had anotia on right side and microtia grade 3 (only rudimentary soft tissue present) on left side and no auditory canal opening was visible bilaterally; there was presence of lower motor neuron type facial nerve palsy on the right side evident from facial asymmetry, absence of forehead wrinkles and inability to close the right eye. Forehead wrinkles and nasolabial folds on the left side were normal. (Figure1).
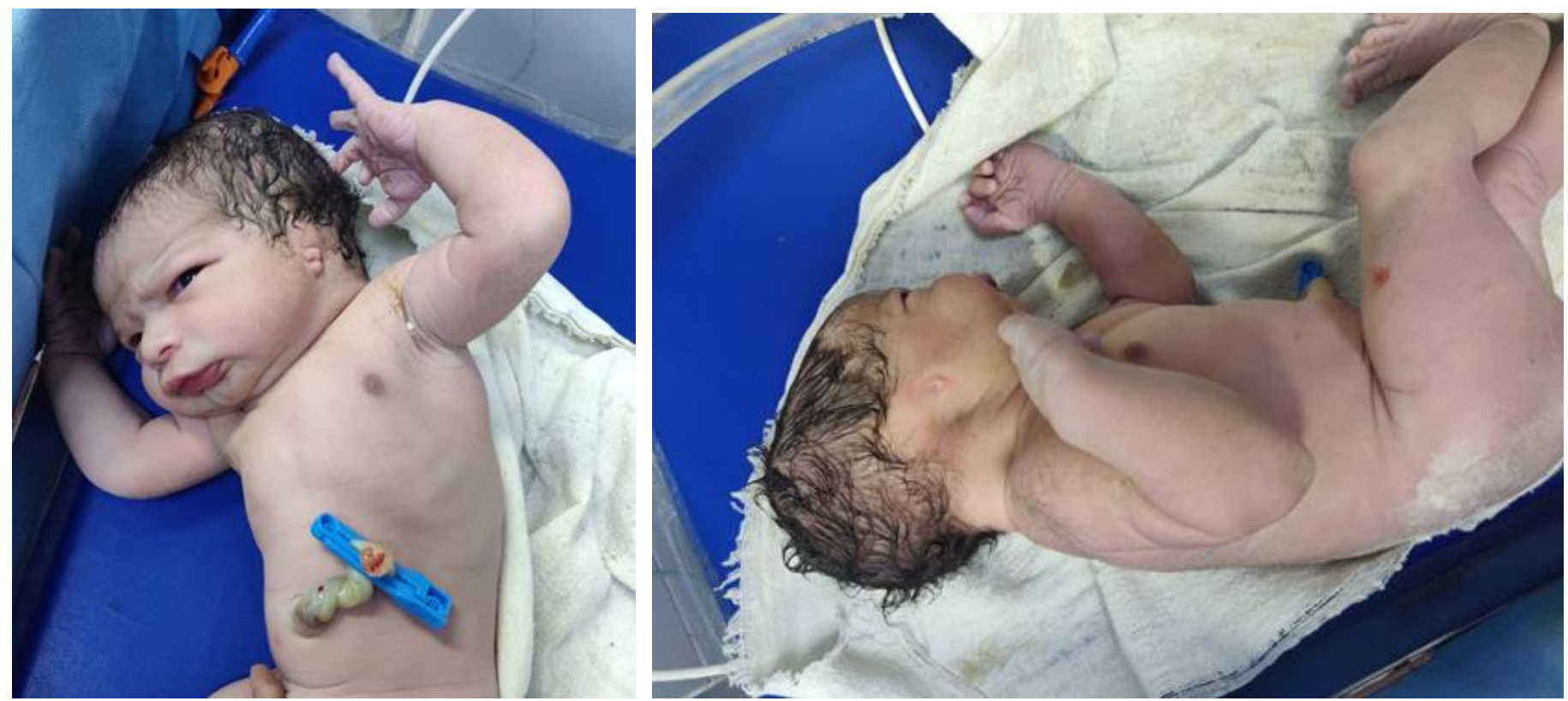

Figure 1: Right side anotia and microtia grade 3 on left side 
As the delivery was spontaneous and uneventful, traumatic delivery and its association with facial nerve palsy as a cause was ruled out. Cardiovascular examination revealed presence of pansystolic murmur. No other congenital anomaly or any other cranial nerve palsy was detected. Other systemic examination was normal. The newborn required oxygen by hood to correct the respiratory distress and intravenous fluids and broad-spectrum antibiotics were started for the baby (Inj cefotaxime 140mg twice daily and inj amikacin 42mg daily). The complete blood count, serum electrolytes and sepsis screen of the newborn was normal. The baby was active, maintaining color and tone, passed both urine and stools within 24 hours. Gradually the baby improved over 2 days, when oxygen was stopped. Nasogastric feeds were initiated and soon the baby was shifted to breastfeed. Ultrasound examination of the abdomen and renal system of the baby was found to be normal. The $2 \mathrm{D} \mathrm{ECHO}$ was suggestive of congenital acyanotic heart disease with a 5-6 $\mathrm{mm}$ osteum secundum (atrial septal defect), a 4-5 mm perimembranous (sub aortic) ventricular septal defect with left to right shunt, and mild tricuspid regurgitation with normal left ventricular systolic function. The MRI brain did not suggest any abnormality. The baby was discharged on $5^{\text {th }}$ postnatal day with advice to follow-up in tertiary centre for further evaluation including genetic analysis which was not available at our institute.

\section{Discussion}

A continuum of congenital anomalies of the auricle is included as microtia with anotia being at the most severe end of the spectrum. Mostly, microtia is unilateral (77-93\%) and if bilateral, is generally associated with other anomalies unre-

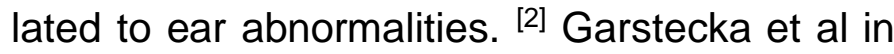
his study of 37 children with external ear malformations found microtia as an isolated anomaly in $46 \%$ children, while $29.7 \%$ were associated with congenital defects of distant organs like the kidney, heart or skeletal system; distant organs were primarily involved when external ear malformations were bilateral. ${ }^{[4]}$ This was true in the study case which had bilateral microtia-anotia with acyanotic heart disease. Kini et al in their study of 98 infants with microtia found renal structural abnormality in $24 \%$; these were seen in $43 \%$ of syndromic children and $21 \%$ of other children. ${ }^{[5]}$ This highlights the importance of ruling out other structural deformities while evaluating a case with microtia. The infant in this case had a normal ultrasound scan of the abdomen and no associated renal or skeletal abnormality was found while congenital heart disease was confirmed on 2D ECHO of the infant.

Etiology of microtia/anotia is poorly understood with both hereditary factors and environmental factors playing a role. Environmental factors found to be associated with microtia-anotia include altitude, maternal obesity, higher maternal age, higher parity, rubella infection during first trimester, pre-pregnancy diabetes, multiple births, low birth weight and maternal use of medications like thalidomide and retinoids. The patient did not have any of these high risk factors but folic acid supplementation which has shown to be protective was not taken by our patient during her antenatal period. [6]

Isolated cases of microtia-anotia are generally autosomal dominant and multifactorial while cases with bilateral anomaly are generally associated with other syndromes including congenital rubella syndrome, Treacher-Collins syndrome, Velo-cardio-facial syndrome, thalidomide or retinoic acid embryopathy, diabetic embryopathy or as a variant of Goldenhar syndrome. The infant in this case had congenital bilateral anotia with unilateral facial paralysis and heart disease, a triad which has been first described by Pearl in 1984. This triad has also been seen as a variant of Goldenhar syndrome or as a teratogenic effect of thalidomide or retinoic acid ingestion in the mother during pregnancy. ${ }^{[7]}$ If thalidomide is ingested between 34 to 38 days after the last menstrual day by the pregnant woman, it disturbs the common pathway in the development of hyoid arch (from which the ear develops), the cardiac primordial and the facial nerve leading to these anomalies. ${ }^{[8]}$ Our patient did not have history of ingestion of any medications other than calcium supplements. 
Goldenhar syndrome (GS), which is mostly sporadic, includes a wide range of clinical manifestations including microtia and facial asymmetry. [9] But, the typical features of GS include mandibular hypoplasia (which is the cause of facial asymmetry), epibulbar dermoids and vertebral anomalies none of which were not present in our case. Velo-cardio-facial syndrome, an autosomal dominant disorder, also consists of asymmetric crying facies and congenital heart disease but it is also associated with palatal and other structural abnormalities including skeletal, cranial and ocular. ${ }^{[10]}$ The facial weakness in these cases is partial and is only apparent when the infant cries but facial asymmetry was apparent in the infant in our case even when he was quiet. (Figure 2) Also, ear abnormalities are not seen in infants with this syndrome which were present in our case.

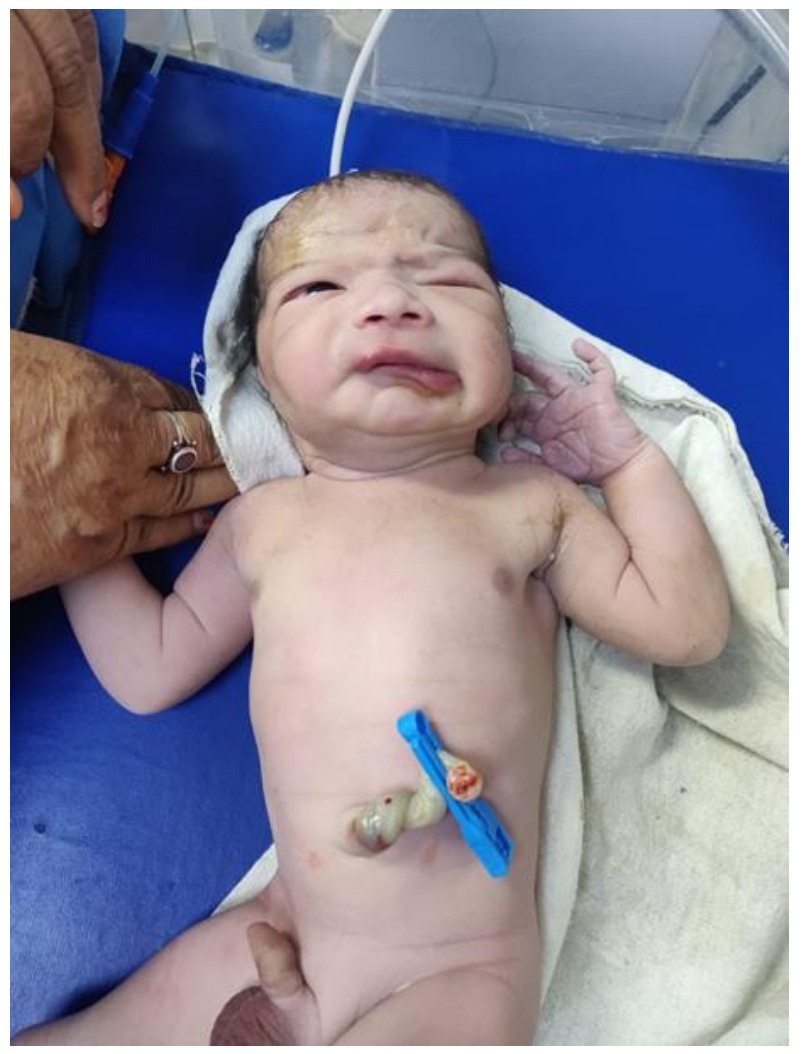

Figure 2: Facial palsy of right side

Microtia can be part of Treacher-Collins syndrome (TCS) which is a rare genetic disorder resulting in congenital craniofacial malformations and is primarily caused by mutation in TCOF 1 gene. ${ }^{[11]}$ The characteristic feature of TCS is involvement of facial bones and it is mostly associated with hypoplastic malars, and mandibular and pharyngeal hypoplasia which were all absent in this case.

Another rare disorder associated with microtia is Nager syndrome which has an autosomal dominant inheritance and is caused by mutation in SF3B4 gene. The main deformities seen are craniofacial including malar and mandibular hypoplasia and microtia and limb anomalies in the form of absent or underdeveloped thumb and radial bone and radioulnar synostosis. Limb deformities which are a cardinal sign of this syndrome were absent in our case. ${ }^{[12]}$

CHARGE syndrome is a rare disorder affecting multiple organ systems and is caused by mutation in the CHD7 gene. It is mnemonic that stands for coloboma, heart defect, choanal atresia, retarded growth, genital hypoplasia and ear abnormalities. The diagnosis is based on the presence of at least one major criterion i.e coloboma, cranial nerve abnormalities (I, VII, VIII, IX $\& X)$, choanal atresia and presence of typical CHARGE ear. ${ }^{\left[{ }^{[3]}\right.}$ Our patient did have asymmetric facial palsy which is just one of the many 
cranial nerves associated with this syndrome. Also, the infant had anotia and not the typical Charge ear which is short, wide and floppy with no ear lobe.

Microtia-anotia which is apparent at birth should alert the treating neonatologist to further evaluate the infant to exclude congenital anomalies of distant organs; this is even more relevant if the condition is bilateral. Early diagnosis is essential not only for appropriate treatment and genetic counseling but also for social and psychological rehabilitation of the child later.

\section{References}

[1] Bartel-Friedrich S. Congenital Auricular Malformations: Description of Anomalies and Syndromes. Facial Plast Surg. 2015; 31(6):567-80.

[2] Luquetti DV, Heike CL, Hing AV, Cunningham ML, Cox TC. Microtia: epidemiology and genetics. Am J Med Genet A. 2012; 158A (1):124-139.

[3] Kaur, M, Kaura, S, Sharma, A \& Showkat R. (2020). Restoration of Microtia by Prosthetic Method: A Case Report. European Journal of Medical and Health Science 2020; 2(4). https://doi.org/10.24018/ejmed.2020.2.4.

[4] Garstecka A, Betlejewski S, Skonieczka K. Microtia: isolated defect of hearing organ, or syndrome forming collection of abnormalities. Otolaryngol Pol. 2008; 62(5):639-42.

[5] Kini S, Barton GW, Carol Liu YC. Renal anomalies and microtia: determining the clinical utility of screening affected children. Int J Pediatr Otorhinolaryngol. 2020 Jun. 133:109957.

[6] Ryan MA, Olshan AF, Canfield MA, et al. Sociodemographic, health behavioral, and clinical risk factors for anotia/microtia in a population-based case-control study. Int J Pediatr Otorhinolaryngol. 2019 Mar 23. 122:18-26.

[7] Kumar S, Ujlayan V, Singh JK, Jha C. Pearl syndrome with Jacob syndrome: A rare association. IJSR 2019; 8(8):25-26.

[8] Lenz W. A short history of thalidomide embryopathy. Teratology 1988 Sep; 38(3):203-15.

[9] Martelli H Jr, Miranda RT, Fernandes CM, et al. Goldenhar syndrome: clinical features with orofacial emphasis. J Appl Oral Sci. 2010; 18(6):646649.

[10] Shprintzen RJ. Velo-cardio-facial syndrome: 30 Years of study. Dev Disabil Res Rev. 2008; 14(1):3-10.

[11] Chang CC, Steinbacher DM. Treacher collins syndrome. Semin Plast Surg. 2012; 26(2):83-90.

[12] Ju-Li-Lin. Nager syndrome: A case report. Pediatrics and Neonatology 2012;53(2);147-150.

[13] van Ravenswaaij-Arts CM, Hefner M, Blake K, et al. CHD7 Disorder. 2006 Oct 2 [Updated 2020 Sep 17]. In: Adam MP, Ardinger HH, Pagon RA, et al., editors. Gene Reviews ${ }^{\circledR}$ [Internet]. Seattle (WA): University of Washington, Seattle; 19932021. Available from:

https://www.ncbi.nlm.nih.gov/books/NBK1117/

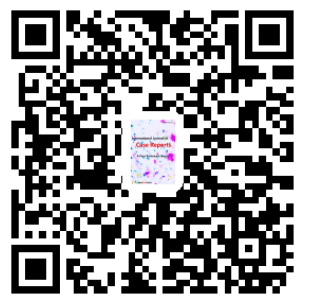

